\title{
Composite alginate microparticles for controlled drug release
}

\author{
A. Hubatová-Vacková ${ }^{1}$, Denisa Lizoňová ${ }^{1}$, A. Pittermannováa ${ }^{1,2}$, V. Tokárová ${ }^{1}$, O. Kašpar ${ }^{1}$, \\ F. Štěpánek ${ }^{1}$
}

1. Department of Chemical Engineering, University of Chemistry and Technology, Technická 3, 16628 Prague

6, Czech Republic.

2. Laboratoire Collö̈des et Matériaux Divisés, ESPCI ParisTech, 10 rue Vauquelin, 75005 Paris, France

\begin{abstract}
:
Microparticles introduced in this work are made of hydrophilic polymers with the ability to incorporate various functional components such as magnetic nanoparticles, liposomes, or enzymes [1,2]. Microfluidics allows us to fabricate such microparticles in sizes comparable to blood cells, which makes them suitable for drug delivery [3]. The advantage is that these microparticles can be used as tiny chemical or biochemical reactors to locally produce and release pharmaceutically active substances, which are highly reactive or unstable, and therefore cannot be delivered using conventional drug formulations. Another advantage of such system is the possibility of active targeting using surface modification by specific ligands. The goal of this project was to prepare, test and analyse alginate particles, starting by encapsulating a model fluorescent dye and aiming to prepare microparticles able to deliver an antimicrobial compound. Therefore, the methods of preparation of hydrogel microparticles, more specifically microfluidics and microencapsulation, as well as the encapsulation of active compounds within liposomes will be presented in this work. Methods of controlled release are studied, in particular the delivery of doxycycline and the on-demand production of resorufin by the reaction of resazurin with ascorbic acid.
\end{abstract}

Keywords: chemical robot; microfluidics; microparticle synthesis; surface modification; targeted drug delivery

\section{References:}

1. Ullrich, M., et al., Encapsulation stability and temperature-dependent release kinetics from hydrogel-immobilised liposomes. Journal of Colloid and Interface Science, 2013. 394: p. 380-385.

2. Hanuš, J., et al., Remotely Controlled Diffusion from Magnetic Liposome Microgels. Langmuir, 2013. 29(13): p. 4381-4387.

3. Pittermannová, A., et al., Microfluidic fabrication of composite hydrogel microparticles in the size range of blood cells. RSC Advances, 2016. 6(105): p. 103532-103540. 